\title{
Olympischer Anzeiger. Plädoyer, den Sport ernst zu nehmen
}

Es gibt eine - wie mir scheint: zu Recht attraktive - sportwissenschaftliche Tradition, den Sport als Seismographen für Zustand und Entwicklungstendenzen der Gesellschaft anzusehen. Diese Tradition verdichtet sich in der Metapher vom Sport als Spiegel der Gesellschaft, die mit wechselnden Konjunkturen insbesondere in der Sportsoziologie und Sportphilosophie beiläufig gebraucht, emphatisch affirmiert oder heftig bestritten wird. Die Figur des Spiegels bzw. Seismographen setzt nämlich streitbar voraus, dass sich der Sport mimetisch auf diejenige Gesellschaft bezieht, in der er praktiziert wird, und dass in diesem mimetischen Bezug sein politischer Gehalt verortet ist. In dieser Tradition drängt sich die Theatermetaphorik auf: die aufgeführte oder dargestellte Gesellschaft (exemplarisch Gebauer u. a. 2004). Der sportliche Wettkampf wird dort als Drama begriffen, aufgeführt auf der Bühne des organisierten Sports, allein seiner eigenen Logik verpflichtet, aber genau dadurch aufzeigend, wie es in der Welt außerhalb dieser Bühne sein ,könnte“ (Aristoteles, Poe: $1451 \mathrm{a}-\mathrm{b})$. Hier fällt das Politische des Sports nicht mit der ihn unvermeidbar begleitenden Sportpolitik zusammen; vielmehr liegt sein politischer Gehalt in seiner Form, die er nicht einfach hat, sondern die es zu wahren gilt - ganz analog dazu, dass der politische Gehalt eines Kunstwerks, der allein in der künstlerischen Form liegt, durch allzu übergriffige Kulturpolitik gefährdet sein mag, aber weder durch Kulturpolitik konstituiert wird noch per se durch sie zerstört wird. Mimetische Bezugnahme heißt beides zugleich: Mit Gumbrecht (2005) ist der Sport eine Präsenz-Kultur, aber gegen ihn ist eine Präsenz-Kultur eine Ausdrucks-Kultur.

Die Metapherngeschichte des Spiegels zeigt diese Logik des Zugleich auf. Etwas im Spiegel sehen heißt, etwas in einem anderen ontologischen Status, nämlich als (Spiegel-)Bild, zu sehen. Etwas als Bild zu sehen, heißt weder, ein Abbild (hier: der Gesellschaft) zu sehen, noch heißt es, etwas so zu sehen, als ob es ein Bild wäre. Man muss zwar, selbstverständlich, jene Voraussetzung mimetischer Bezugnahme nicht mitmachen, aber es ist, gemessen an dieser Metapherngeschichte, ein gedanklicher Fehler, sie deshalb nicht mitzumachen, weil man Spiegel-Sehen nicht von Abbildungen einerseits und Vor-Spiegelungen andererseits unterscheiden kann (ausführlicher Schürmann 1999, Kap. 4.1). 
Für die Epoche der klassischen Moderne kann man nun in der Tat sagen, dass der olympische Sport eine solche Aufführung der modernen Gesellschaft ist. Nimmt man ihn, methodisch begründet, als Prototyp des Sports der Moderne (Schürmann 2010), dann kann man in ihm die spielerische Inszenierung des Grundprinzips dieser Moderne sehen. Im bürgerlich-revolutionären Bruch mit der Vormoderne lautet das Versprechen moderner Gesellschaften, dass die soziale Stellung des Einzelnen nicht qua Geburt, Natur oder Gott vor-gegeben festliegt, sondern von Jedem und Jeder veränderbar ist - letztlich deshalb, weil Alle als freie Mitautoren der Ordnung des sozialen Miteinander gelten. Das freilich kann nur bei aktiver Sozialpolitik gelingen, die für die Herstellung gleicher Startchancen sorgt und diese gewährleistet, denn „wenn ein Riese und ein Zwerg auf derselben Straße gehen, so wird jeder Schritt, den sie beide tun, dem Riesen einen erneuten Vorsprung verschaffen" (Rousseau 1755: $69 \mathrm{f}$.). Die zentrale Errungenschaft der bürgerlichen Revolutionen ist das Versprechen des Schutzes der unaustauschbaren Einmaligkeit, sprich: der Würde jedes und jeder Einzelnen. Dies wird durch die Umstellung aller gesellschaftlichen Verhältnisse auf prinzipielle Vermitteltheit erreicht. Personen begegnen sich nicht mehr direkt, nur willkürlich geschützt von Angesicht zu Angesicht, sondern prinzipiell vermittelt als Citoyens, als Staatsbürger, also prinzipiell vermittelt durch ihr wechselseitiges Sich-Anerkennen als Person gleicher Rechte. Oder auch: Die Menschenrechtserklärungen postulieren keine Gleichheit von Menschen, sondern schützen - ganz im Gegenteil - deren anerkannte Verschiedenheit und je eigene Entwicklungsmöglichkeiten durch fragloses Zusprechen gleicher Rechte.

Genau dieses Prinzip der Staatsbürgerschaft wird in einem Wettkampf des olympischen Sports spielerisch inszeniert. Ein für die jeweilige Sportart konstitutives Regelwerk platziert sportliches Tun in eine eigene Welt - ein Boxkampf ist prinzipiell etwas anderes als eine durch Regularien zivilisierte Straßenprügelei, aktive Sportpolitik hat für die Herstellung und Wahrung gleicher Startchancen all derjenigen zu sorgen, die zu einem Wettkampf antreten (wollen), damit auf dieser Basis tatsächlich die individuelle Leistung über Sieg und Niederlage entscheiden kann. Gegen einen von vornherein unterlegenen Gegner zu gewinnen, ist sozusagen keine Kunst, und jedenfalls kein sportlicher Sieg. Die sportliche Form zu wahren, heißt, sie vor äußeren Übergriffen zu bewahren und die eigene innere Logik aufrecht zu erhalten - vergleichbar damit, dass eine Propagandaschrift keine künstlerische Form ist. Ein Sieg jedoch, so das Spiel des Olympismus, ist nur eine Hierarchisierung auf Zeit, die sich ihrerseits wiederum höchstens durch individuelle Leistung verstetigen soll - nach dem Spiel ist vor dem Spiel, und schon beim nächsten Aufeinandertreffen bestehen wieder gleiche Startchancen. In diesem Sinne ist Fairness das Grundprinzip des olympischen Sports, wobei Fairness nichts weiter heißt als eben dies: Die 
aktive Herstellung und Wahrung der Offenheit des Ausgangs des Wettkampfs. Menschen kämpfen dort nicht auf Teufel komm raus um Überlegenheit, sondern sportliche Parteien in all ihrer Individualität und Unterschiedlichkeit treten als Gegner gegeneinander an, die sich auf die Gleichheit ihrer Ausgangsstärke, also auf die Offenheit des Ausgangs verpflichtet haben (Schürmann 2006, 2011).

Beinahe überall auf der Welt wird die Würde des Menschen angetastet, und beinahe überall im heutigen Olympismus wird die Offenheit des Ausgangs eines sportlichen Wettkampfs auszuhebeln versucht, etwa durch Doping, Korruption oder Wettbetrug. Das als solches spricht weder gegen die Menschenrechtserklärungen noch gegen die olympische Charta - im Gegenteil. Ohne geltendes Völkerrecht wäre Folter lediglich ein Verstoß gegen die Sondermoral bestimmter Kulturen, nicht aber ein sanktionierbarer Rechtsverstoß; ohne die olympische Charta wären Spielabsprachen lediglich ein Problem der Individualmoralen der sogenannten schwarzen Schafe, nicht aber ein Bruch der wechselseitigen Verpflichtung auf den Witz des Sports.

Nun ist aber ganz offenkundig, dass die genannten Verstöße gegen die Fairness keineswegs einzelne, mehr oder weniger häufig auftauchende Ausnahmen sind, sondern zum System des heutigen Olympismus gehören. Am Beispiel des Doping ist das lange bekannt und vielfach herausgestellt worden (exemplarisch Bette/Schimank 1995). Doping ist nicht nur ein systemisches Problem, sondern zu diesem systemischen Problem gehört dazu, dass es von einem Großteil der Beteiligten Funktionäre, Publikum, Medien, weite Teile der Sportwissenschaft - gerade nicht als systemisches Problem dargestellt wird, sondern weiterhin als ein Problem zu bestrafender schwarzer Schafe individualisiert wird. Die derart Agierenden können derweil ihre eigenen Schäfchen ins Trockene bringen. Wenn diese Diagnose zutrifft, wäre der Olympismus ein System, das systematisch sein eigenes Versprechen bricht. Wie soll man das erklären?

Das moderne Prinzip und Versprechen der Staatsbürgerschaft kann, im Prinzip, in ganz unterschiedlichen Gesellschaftsformationen realisiert werden. Man sagt wohl nichts Falsches oder gar Böses, wenn man konstatiert, dass dieses Prinzip im Westen hegemonial in der Formation der bürgerlichen Gesellschaft realisiert ist. Das Prinzip der Staatsbürgerschaft ist, formelhaft, das Prinzip der Republik: die Macht aller Citoyens über alle Citoyens - in der bürgerlichen Gesellschaft ist dieses Prinzip realisiert als, ebenso formelhaft, Herrschaft der Bourgeois über die Citoyens. Versprochen wurde uns die Freiheit von Religion - bekommen haben wir die Religionsfreiheit (Marx 1844, MEW 1: 369; Stirner 1844: 115). Versprochen wurde uns Gerechtigkeit zum Schutz unserer Würde - bekommen haben wir Leistungs- 
gerechtigkeit. Eingedenk dessen wäre es seltsam, wäre der Olympismus, gedacht als Spiel der Citoyens, nicht ein Unternehmen unter dem Dach der Bürger.

Es gibt einen intrinsischen Konflikt zwischen Leistungsgerechtigkeit und Würde. Vormodern war Würde ein Leistungsbegriff (dignitas): Denjenigen Personen (oder Institutionen), denen Würde zukam, kam sie aufgrund eines spezifischen Merkmals, einer Besonderheit, einer erbrachten oder zu erbringenden Leistung zu. Wir kennen diese Bedeutung noch heute etwa in der Rede von der „Würde eines Amtes“. Mit den bürgerlichen Revolutionen und den Erklärungen der Menschenrechte ändert sich das grundlegend: Würde ist nun, so die berühmte Kantsche Unterscheidung von Preisen, eine Achtung in der Weise, dass sie denjenigen, denen sie zukommt, fraglos und kategorisch zukommt, ohne weitere Bedingung, ohne zu erbringende Aufnahmeprüfung in den Kreis der Würdigen. Das geht damit einher, dass fraglos allen Menschen diese fraglose, bedingungslose Würde zukommen soll. Gerecht ginge es daher in der Gesellschaft dann zu, wenn Jede und Jeder seine oder ihre Eigentümlichkeit einschließlich der damit verknüpften Entwicklungsmöglichkeiten pflegen könnte, und wenn Jede und Jeder seinen oder ihren Anteil dazu beiträgt, dass dies für alle möglich ist. Übersetzt in heutige Debatten: Bedingungsloses Grundeinkommen für alle; unter der Bedingung, dass Jede und Jeder zu einem Beitrag für das Gemeinwohl herangezogen werden kann. „Die Verkürzung des Arbeitstages ist die Grundbedingung.“ (Marx 1894, MEW 25: 828) - Das klingt dann doch zu sehr nach paradiesischen Zuständen im Nirgendwo. An dieser Stelle jedenfalls ertönen notorisch die Unkenrufe, die vor den Trittbrettfahrern, neudeutsch: freerider, warnen. Und genau darin liegt die kleine, aber wirksame bürgerliche Verschiebung des Prinzips. Eben nicht: „Jede und jeder nach ihren und seinen Bedürfnissen!" (und damit das möglich wird, hat jede ihre und jeder seine Verpflichtungen), sondern aus der versprochen unbedingten Gerechtigkeit wird eine bedingte: „Jeder nach seiner Leistung!“ In den Genuss der Gerechtigkeit kommt man in der bürgerlichen Gesellschaft dann und nur dann, wenn man etwas leistet. Und daraus folgt: Es ist nicht mehr fraglos, sondern muss von Humanisten eigens begründet werden, dass auch denjenigen, die noch nicht, nicht mehr oder aktuell nicht leisten (Säuglinge, Alte, Koma-Patienten etc.), Würde zukommt. Auch in der bürgerlichen Philosophie wird daher das Problem der „Grenzen der Gerechtigkeit“ diskutiert (etwa Nussbaum 2010). Angesichts von drei drängenden Problemen - „Behinderung, Nationalität und Spezieszugehörigkeit“ - wird zwar für eine „Revision der theoretischen Struktur selbst“ (ebd.: 14) geworben, aber das ist de facto eine Reparaturarbeit an Rawls. Es werden Gründe gegeben, warum auch die Nicht-Leistenden zu uns gezählt werden sollen - das bereits zur Leistungsgerechtigkeit verschobene Prinzip wird also ex negativo bestätigt. Es ist nämlich ein logischer Unterschied, ob 
erwiesen werden soll, dass es mehr Kandidaten gibt, die die Bedingungen erfüllen, in den Gesellschaftsvertrag aufgenommen zu werden, oder ob man betont, dass Personen bedingungslos dazugehören. Der Verrat am Versprechen bedingungsloser Würde ist im Gezänk zwischen Liberalisten, Kommunitaristen und Kompromisslern auf Dauer gestellt und übertönt.

Unter den Bedingungen der Herrschaft von Leistungsgerechtigkeit wundert es daher nicht, dass es entsprechend zu einem intrinsischen Konflikt zwischen staatsbürgerlich-zivilgesellschaftlichem Sport und Olympismus der Bürger kommt. Verstöße gegen die Fairness gehören systemisch zum Olympismus der Bürger hinzu, und das Beschwören von Fairness kommt komplementär/additiv/bemäntelnd hinzu. Das Leistungsprinzip des zivilgesellschaftlichen Sports gerät dort zum Erfolgsprinzip, garniert durch Sonntagsreden respektive durch eine Sportpolitik, die den schönen Schein wahren will. Um nur einige Symptome zu nennen: Es gibt keine Kultur der Niederlage (Ränsch-Trill 2006); es gibt keine Gewaltenteilung im IOC, das hier eher dem Vatikan vergleichbar ist als einer demokratischen zivilgesellschaftlichen Organisation (Rittberger/Boekle 1997); es herrscht vielfach offene Gleichgültigkeit gegenüber den Möglichkeiten tatsächlicher Partizipation am olympischen Sport (niemand in der olympischen Bewegung findet auch nur bemerkenswert, dass es niemals marokkanische Rodlerinnen gibt); die neu ins Leben gerufenen olympischen Jugendspiele stehen völlig zu Recht - ein Blick auf die Internetseiten genügt - im Verdacht, nichts weiter als die frühe Einübung des Erfolgsprinzips zu sein, denn gerade auch dort wird die sportliche Leistung am Erfolg, sprich am Primat der Medaillen dingfest gemacht.

Um Missverständnisse zu vermeiden: Die Betonung liegt auf der Differenz von Leistungs- und Erfolgsprinzip. Das Leistungsprinzip ist für den modernen Sport unverzichtbar. Dazu gäbe es zweifellos sehr viel zu sagen und zu präzisieren. Aber es meint hier zunächst nur, dass der moderne Sport als Wettkampf organisiert ist. Das wiederum hat zwei Aspekte, die hier wichtig und für das Argument hinreichend sind, nämlich dass es sich a) um ein (mit Simmel 1903) prinzipiell indirektes Verhältnis der Konkurrenz handelt, das b) in spielerischer Weise zu vollziehen ist. Gestalteter Wettkampf heißt daher nun einmal gerechter Leistungsvergleich, und darin liegt nicht als solches schon ein Verrat am Versprechen moderner Staatsbürgerschaft. Zwar gilt für die Gesamtgesellschaft, dass die herrschende Leistungsgerechtigkeit ein Verrat am Prinzip ist, da es eine bedingte Gerechtigkeit ist. Die Beteiligung am Olympismus dagegen ist freiwillig, und eine fragliche Gerechtigkeit dort hat insofern von vornherein die Form: „Falls man teilnimmt, dann ...“. Und insofern wissen alle, worauf sie sich einlassen: Falls sie teilnehmen, dann heißt Gerechtigkeit dort gerechter Leistungsvergleich. Gleichwohl besteht der Witz des Sports gerade 
nicht darin, alles für den Sieg zu geben. Es geht gerade nicht darum, auf Teufel komm raus Erster zu sein - denn dann würde man beim Marathonlauf eine Abkürzung nehmen (und versuchen, sich nicht erwischen zu lassen). Vielmehr geht es darum - und nur das zählt als sportliche Leistung -, den Sieg in einer bestimmten, nämlich fairen Art und Weise zu erringen. Man soll olympisch nicht alles, sondern das Beste für den Sieg geben. Fairness ist der konstitutive Modus des sportlichen Gewinnenwollens, und gerade nicht etwas, das additiv, also lediglich regulativ, zum Erfolgswillen hinzutritt (Schürmann 2008).

Die Vergleichbarkeit - das sozusagen Seismographische - liegt also in der Selbigkeit der Logik: So, wie zur bürgerlichen Leistungsgerechtigkeit die versprochene Würde Aller nur additiv-bedingterweise hinzukommt - und entsprechend empirisch-faktisch die Würde der Nicht-Leistenden systemisch durchs Raster der Leistungsgerechtigkeit hindurch fällt -, genau so kommt zum bürgerlichen Erfolgsprinzip die versprochene Fairness nur additiv-beschwörend hinzu - und entsprechend gilt im olympischen Sport empirisch-faktisch das, was Lenk das 11. Gebot genannt hat: Du sollst Dich nicht erwischen lassen!

Ob Fairness ein konstitutiver Modus oder lediglich ein Regulativ ist, sprich: ob im Sport das Leistungs- oder aber das Erfolgsprinzip praktiziert wird, ist keine Frage der Moral der beteiligten Individuen, sondern eine Frage der Organisation der real praktizierten Wettkämpfe. Freilich kommt es vor, dass die Moral in verderbten Organisationen verdirbt. Die oben aufgezeigten Symptome sprechen eine deutliche Sprache in Bezug auf den heutigen Olympismus. Was demgegenüber ein republikanischer Olympismus sein könnte, kann man sich nicht vorab ausmalen. Wohl kann man den heutigen Olympismus an jenem, seinem Versprechen messen. Vielleicht beginnt er dann ja, dereinst bei hinreichend Resonanz, zu tanzen (Marx 1844, MEW 1: 381).

Wofür das spricht? Nun, ganz einfach: Falls man den Sport als Seismographen der Gesellschaft begreift, dann muss man den olympischen Anzeiger zu lesen verstehen. Dann kann man gleichsam sehen, dass jede Gesellschaft denjenigen Sport praktiziert, der ihr entspricht und den sie folglich verdient. Und da hilft es nicht, den Boten zu schlagen - falls man denn überhaupt für problematisch hält, was er anzeigt. 


\section{Literatur}

Aristoteles (Poe), 1982: Poetik. Übersetzt und herausgegeben von Manfred Fuhrmann, Stuttgart.

Bette, Karl-Heinrich/Schimank, Uwe, 1995: Doping im Hochleistungssport, Frankfurt a. M.

Gebauer, Gunter/Alkemeyer, Thomas/Boschert, Bernhard/Flick, Uwe/Schmidt, Robert, 2004: Treue zum Stil. Die aufgeführte Gesellschaft, Bielefeld.

Gumbrecht, Hans Ulrich, 2005: Lob des Sports, Frankfurt a. M.

Marx, Karl (MEW), 1844 ff./1956 ff.: Marx-Engels-Werke. Diverse Bände, Berlin. Nussbaum, Martha Craven, 2010: Die Grenzen der Gerechtigkeit. Behinderung, Nationalität und Spezieszugehörigkeit, Berlin.

Ränsch-Trill, Barbara, 2006: Siegen und Verlieren. Zum Desiderat einer Kultur der Niederlage im Sport, in: Leipziger Sportwissenschaftliche Beiträge 47 (2), 171-179.

Rittberger, Volker/Boekle, Henning, 1997: Das Internationale Olympische Komitee - eine Weltregierung des Sports?, in: Ommo Grupe (Hrsg.), Olympischer Sport. Rückblick und Perspektiven, Schorndorf, 127-155.

Rousseau, Jean-Jacques, 1755/2005: Abhandlung über den Ursprung und die Grundlagen der Ungleichheit unter den Menschen. Herausgegeben von Philipp Rippel, Stuttgart.

Schürmann, Volker, 1999: Zur Struktur hermeneutischen Sprechens. Eine Bestimmung im Anschluß an Josef König, Freiburg i. Br./München.

Schürmann, Volker, 2006: „Die schönste Nebensache der Welt“. Sport als Inszenierung des Citoyen, in: Deutsche Zeitschrift für Philosophie 54 (3), 363-382.

Schürmann, Volker, 2008: Zur Normativität des Sports, in: Spectrum der Sportwissenschaften 20 (1), 45-63.

Schürmann, Volker, 2010: Prototypen. Zur Methodologie einer Hermeneutik des Sports, in: Sport und Gesellschaft 7 (3), 55-64.

Schürmann, Volker, 2011: Olympische Spiele im Spiegel der Menschenrechte, in: Elk Franke (Hrsg.), Ethik im Sport, Schorndorf, 61-73.

Simmel, Georg, 1903/1995: Soziologie der Konkurrenz, in: ders., Gesamtausgabe in 24 Bänden. Band 7: Aufsätze und Abhandlungen 1901-1908, Frankfurt a. M., 221-246.

Stirner, Max, 1844/2009: Der Einzige und sein Eigentum. Ausführlich kommentierte Studienausgabe. Herausgegeben von Bernd Kast, Freiburg i. Br./München. 
Korrespondenzanschrift:

Prof. Dr. Volker Schürmann

Deutsche Sporthochschule (DSHS) Köln

Institut für Pädagogik und Philosophie

Am Sportpark Müngersdorf 6

50933 Köln

E-Mail: v.schuermann@dshs-koeln.de

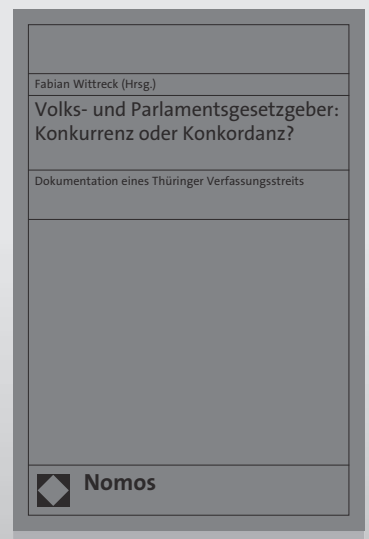

WT
Volks- und Parlamentsgesetzgeber: Konkurrenz oder Konkordanz?

Dokumentation eines Thüringer

Verfassungsstreits

Herausgegeben von Fabian Wittreck

2012, 215 S., brosch., 58,- $€$

ISBN 978-3-8329-7252-3

Kann das Parlament vom Volk beschlossene Gesetze wieder aufheben? Kann es während eines laufenden Volksbegehrens diesem die Grundlage entziehen, indem es Änderungen am Antragsgegenstand vornimmt? Die im vorliegenden Band dokumentierten Gutachten zum Streit um ein Thüringer Volksbegehren stellen die bislang umfassendsten Analysen des Verhältnisses von Volks- und Parlamentsgesetzgeber dar, das zukünftig häufiger die Gerichte beschäftigen dürfte. 